\title{
CONSIDERAÇÕES TAXONÔMICAS E NOVAS COMBINAÇÕES EM ARDISIA SWARTZ (MYRSINACEAE) DO SUDESTE DO BRASIL ${ }^{1}$
}

\author{
Luís Carlos Bernacci ${ }^{2}$ \\ Sigrid L. Jung-Mendaçolli²,3
}

Recebido em 24/06/1999. Aceito em 26/04/2000

\begin{abstract}
RESUMO - (Considerações taxonômicas e novas combinações em Ardisia Swartz (Myrsinaceae) do sudeste do Brasil). Os tipos de placentação e de pré-floração têm sido utilizados para a distinção dos gêneros Ardisia Swartz, ao qual tem sido atribuído placentação plurisseriada e pré-floração imbricada ou quincuncial, e Stylogyne A.DC., ao qual tem sido atribuído placentação unisseriada e pré-floração contorta. Entretanto, a análise de dez espécies destes gêneros revelou a inconsistência destes caracteres. Também foi constatada a impossibilidade de observar diferenças no tipo de placentação em espécimes com poucos óvulos. Propõe-se, portanto, o restabelecimento da circunscrição de Ardisia, incluindo o gênero Stylogyne, conforme adotado por Miquel, em 1856 e Handro, em 1969. Desta forma, Ardisia ambigua Mart. é o nome válido para S. ambigua (Mart.) Mez e Ardisia martiana Miq. o é para S. laevigata (Mart.) Mez. Duas novas combinações são necessárias: Ardisia depauperata (Mez) Bernacci \& Jung-Mendaçolli e Ardisia warmingii (Mez) Bernacci \& Jung-Mendaçolli.
\end{abstract}

Palavras-chave - Ardisia, Stylogyne, Myrsinaceae, Sudeste do Brasil, taxonomia

\begin{abstract}
Taxonomic considerations and new combinations in Ardisia Swartz (Myrsinaceae) from Southeastern Brazil). Placentation and aestivation have traditionally been used as diagnostic features to separate the genera Ardisia Swartz and Stylogyne A.DC. While Ardisia has pluriseriate placentation and imbricate or quincuncial aestivation, Stylogyne has uniseriate placentation and contorted aestivation. However, careful examination of ten species of these genera revealed the inconsistency of these characters in the distinction of the two genera. Also, we noticed the impossibility of observation of placentation types in few-ovuled specimens. Therefore, we propose the re-establishment of the circumscription of Ardisia adopted by Miquel, in 1856, and Handro, in 1969 which includes the genus Stylogyne. In this way Ardisia ambigua Mart. is the valid name for S. ambigua (Mart.) Mez as Ardisia martiana Miq. is for S. laevigata (Mart.) Mez. Two new combinations are necessary: Ardisia depauperata (Mez) Bernacci \& Jung-Mendaçolli and Ardisia warmingii (Mez) Bernacci \& Jung-Mendaçolli.
\end{abstract}

Key words - Ardisia, Stylogyne, Myrsinaceae, Southeastern Brazil, taxonomy

1 Incluso no projeto "Flora Fanerogâmica do Estado de São Paulo" (Auxílios FAPESP, CNPq)

2 Centro de Recursos Genéticos Vegetais e Jardim Botânico, Instituto Agronômico, C. Postal 28, CEP 13001-970, Campinas, SP, Brasil. e-mail: bernacci@barao.iac.br

3 Bolsa de Produtividade em Pesquisa CNPq 


\section{Introdução}

Ardisia Swartz, com 400-500 espécies (Cheih \& Pipoly 1996 apud Pipoly 1996), é o maior gênero das Myrsinaceae (Pipoly 1991). Tem distribuição tropical, mas não ocorre na África e é raro na Austrália (Mabberley 1987).

Mez (1902) distinguiu a tribo Ardisiae da tribo Myrsineae pela placentação plurisseriada na primeira e uniseriada na segunda. De acordo com Pipoly (1996), a tribo Ardisieae necessita de revisão em escala mundial, porque a delimitação genérica é controversa e inconsistente entre as regiões do planeta; enquanto no Neotrópico a tribo tem sido segregada em vários gêneros, no Paleotrópico, espécies de vários gêneros têm sido sinonimizadas em Ardisia. Miquel (1856) incluiu Stylogyne na circunscrição de Ardisia.

Os gêneros Stylogyne A.DC. e Geissanthus Hooker.f., ambos da tribo Myrsineae, freqüentemente são confundidos com Ardisia (Ricketson \& Pipoly 1997). Cabe ressaltar que têm sido descritas espécies de Ardisia com poucos óvulos (3-5) em que a placentação distribuise em hélice, aparentando uma única série (Pipoly 1996). Por outro lado, Stylogyne pode apresentar placentação bisseriada (Ricketson \& Pipoly 1997), o que também ocorre em Ardisia (Pipoly 1996).

Ricketson \& Pipoly (1997) consideraram que Stylogyne tem distribuição Neotropical e cerca de 60 espécies e pode ser distinto de Ardisia por apresentar cálice e corola contortos e por diferenças na placentação. Entretanto, Pipoly (1996) assumiu a ocorrência de corola contorta em Ardisia.

Durante o estudo das Myrsinaceae da Flora Fanerogâmica do Estado de São Paulo, Brasil, verificou-se que tanto espécies de Ardisia quanto de Stylogyne são referidas para o sudeste do Brasil. Espécies destes gêneros foram examinadas no intuito da verificação da delimitação genérica e correta identificação.

\section{Material e métodos}

Foram analisados materiais de espécies exóticas cultivadas e/ou nativas no Estado de São Paulo e da Área de Proteção Ambiental (APA) Cairuçu (Parati, RJ), depositados nos principais herbários paulistas (BAUR, BOTU, ESA, HRCB, IAC, ISA, SP, SPF, SPFR, SPSF, UEC e UNBA), nos maiores herbários nacionais ( $R$, $\mathrm{RB}$ ), ou em outras coleções (FCAB, HB).

Dos materiais analisados, identificados com base nas delimitações apresentadas por Mez (1902), cinco espécies eram nativas de São Paulo e/ou da APA de Cairuçu:

Ardisia guyanensis (Aubl.) Mez, que se distribui de Trinidad Tobago à Bolívia e Brasil (região norte até $\mathrm{SC}$ );

Stylogyne ambigua (Mart.) Mez, que se distribui da Colômbia à Bolívia e Brasil (MS, MG, $\mathrm{SP})$;

Stylogyne depauperata Mez, exclusiva do Brasil (RJ e SP);

Stylogyne laevigata (Mart.) Mez, exclusiva do Brasil (RJ e SP);

Stylogyne warmingii Mez, exclusiva do Brasil (MG e SP).

Outras cinco espécies eram exóticas:

Ardisia compressa Kunth, que se distribui de Belize ao Equador, sendo cultivada em São Paulo;

Ardisia crispa (Thunb.) A.DC., que se distribui pelo Japão, Formosa, Himalaia e Oceania, sendo cultivada em São Paulo;

Ardisia humilis Vahl, que se distribui pelo Havaí e Oceania, sendo cultivada em São Paulo;

Ardisia solanacea Roxb., que se distribui pelo Sri Lanka, Havaí e Himalaia, sendo cultivada em São Paulo;

Ardisia venosa Mast., que se distribui do México a El Salvador.

De Ardisia compressa e de Stylogyne ambigua foram obtidos materiais frescos, que foram analisados antes da herborização. Alguns 
Tabela 1. Características de espécies atribuídas a Ardisia e Stylogyne nativas do Estado de São Paulo e de outras utilizadas para comparação. n número de flores observadas; masc. - flor estaminada.

\begin{tabular}{|c|c|c|c|c|c|c|c|}
\hline \multirow[t]{2}{*}{ Espécie } & \multirow[t]{2}{*}{ Material } & \multirow[t]{2}{*}{ Herbário } & \multicolumn{2}{|c|}{ Corola } & \multicolumn{2}{|c|}{ Ovário } & \multirow[t]{2}{*}{$\mathrm{n}$} \\
\hline & & & Lobos & Tipo & Óvulos & Placentação & \\
\hline \multirow[t]{3}{*}{ Ardisia compressa Kunth } & Bernacci 2659 & IAC & 5 & Contorta & Muitos & Mais de 2-seriada & 5 \\
\hline & Lorenzi s.n. & IAC 32348 & 5 & Contorta & Muitos (18) & Mais de 2-seriada & 2 \\
\hline & Souza s.n. & IAC 22283 & 5 & Contorta & Muitos & Mais de 2-seriada & 7 \\
\hline A. crispa (Thunb.) A.DC. & Fonegra s.n. & IAC 33310 & 5 & Contorta & 3 & Unisseriada & 2 \\
\hline \multirow[t]{4}{*}{ A. guyanensis (Aubl.) Mez } & Black 48-3324 & IAC & 4 & Contorta & 9 & Bisseriada & 2 \\
\hline & Franciosi et al. 2 & ESA, IAC, UEC & 4 & Contorta & 5 & Unisseriada & 1 \\
\hline & Jung 365 \& Barros & $\mathrm{IAC}, \mathrm{SP}$ & 4 & Contorta & 3 & Unisseriada & 1 \\
\hline & Melo 982 \& Correa & SP & 4 & Contorta & $2-3$ & Unisseriada & $4^{*}$ \\
\hline A. humilis Vahl & Arbocz S.n. & IAC 32349 & 5 & Contorta & Muitos (23) & Mais de 2-seriada & 1 \\
\hline A. solanacea $\operatorname{Roxb}$ & Pickel s.n. & SPSF 3520 & 5 & Contorta & Muitos & Mais de 2-seriada & 2 \\
\hline A. venosa Mast. & Tucker 1243 & IAC & 4 & Contorta & 5 & Unisseriada & 3 \\
\hline \multirow[t]{6}{*}{ Stylogyne ambigua (Mart.) Mez } & Bernacci 1282 & ESA, UEC & 5 & Imbricada & 4 & Unisseriada & 3 \\
\hline & Bernacci 24502 & ESA, UEC & 5 & Contorta & $3-4$ & Unisseriada & 5 \\
\hline & Correa 451 & BAUR, IAC, UEC & 5 & Contorta & 4 & Unisseriada & 2 \\
\hline & Dedecca \& Santoro s.n. & IAC 33643 & 5 & Imbricada & $2-3$ & Unisseriada & 2 \\
\hline & Kuhlmann 382 & IAC, SP & 5 & Contorta & 3 & Unisseriada & 7 \\
\hline & Schuster s.n. & IAC 37817 & 5 & Contorta & 4 & Unisseriada & 4 \\
\hline \multirow[t]{3}{*}{ S. depauperata $\mathrm{Mez}$} & Glaziou 14546 & $\mathrm{RB}$ & 4 & Contorta & - & - & 2(masc.) \\
\hline & Mattos 15749 \& Mattos & SP & 4 & Contorta & $3-4$ & Unisseriada & $1 \#$ \\
\hline & Nadruz 658 & $\mathrm{IAC}, \mathrm{RB}$ & $4(-5)$ & Contorta & - & - & 3(masc.) \\
\hline \multirow{2}{*}{ S. laevigata (Mart.) Mez } & Godoy 239 & SP & 5 & Contorta & $1-2$ & Unisseriada & 3 \\
\hline & Pastore 627 \& Baitello & SP & 5 & Contorta & $4-5$ & Unisseriada & 2 \\
\hline \multirow[t]{3}{*}{ S. warmingii $\mathrm{Mez}$} & Bertoni 11613 & UEC & 4 & Contorta & 4 & Unisseriada & 1 \\
\hline & Jung-Mendaçolli 772 & IAC & 4 & Contorta & 4 & Unisseriada & 1 \\
\hline & Matthes 24046 & UEC & 4 & Contorta & 4 & Unisseriada & 2 \\
\hline
\end{tabular}

* outras três flores observadas eram estaminadas; \# uma outra flor observada era estaminada 
materiais-tipo e ilustrações de dissecções destes foram observados através de fotografias obtidas do Field Museum - USA (F).

Os materiais herborizados foram reidratados para posterior dissecção. As observações foram feitas sob microscópio estereoscópico em diferentes ampliações.

\section{Resultados e discussão}

Todas as espécies (Tab. 1), tanto atribuídas a Ardisia quanto a Stylogyne, apresentaram corola contorta e em dois espécimes (Bernacci 1282 - IAC e Dedecca \& Santoro s.n. - IAC 33643) de S. ambigua (Mart.) Mez, a corola mostrou-se imbricada. Não foi possível observar diferenças no tipo de placentação entre espécies atribuídas a Stylogine ou Ardisia, com poucos óvulos (placentação unisseriada - Tab. 1). Em A. guyanensis (Aubl.) Mez, o número de óvulos variou de três a nove e a placentação mostrou-se bisseriada no material Black 483324 (IAC) ou unisseriada (outros materiais da espécie - Tab. 1). Através da foto da exsicata de A. guyanensis (Ule 704 - F) foi possível observar que, na ilustração que acompanha a exsicata, os óvulos (4-5) estão distribuídos alternadamente, com ligeiro desnível.

Em função da análise realizada e pela comparação com a literatura, não foram detectados elementos que possibilitassem distinguir os dois gêneros. Assim sendo, a circunscrição de Ardisia deve incluir Stylogyne, tal como tratado por Miquel (1856) e Handro (1969).

Desta forma:

Ardisia ambigua Mart., Flora 24, beibl. 2: 63. 1841, é o nome válido correspondente a Stylogyne ambigua (Mart.) Mez in Mart. Pflanzenr. 9: 266. 1902.

Ardisia martiana Miq. in Mart. Fl. brasil. 10: 285 , t. 30.1856 , é o nome válido para Stylogyne laevigata (Mart.) Mez in A. Engler Pflanzenr. 9: 266. 1902. Icacoera laevigata Mart., utilizado como basiônimo de $S$. laevigata, por Mez (1902), é um nomen nudum. Não foi efetivamente publicado e quando Miquel (1856) descreveu $A$. martiana citou-o como sinônimo.

Duas combinações novas são necessárias:

Ardisia depauperata (Mez) Bernacci \& Jung-Mendaçolli. Stylogyne depauperata Mez in A. Engler, Pflanzenr. 9: 277. 1902. Tipo: Brasil, Rio de Janeiro, Beyrich (n.v.); Brasil, Rio de Janeiro, floresta da Tijuca, 29/XII/1869, fl. masc., Glaziou 4073 (síntipo: B, destruído; foto $\mathrm{F}$, IAC!); Brasil, Rio de Janeiro, Glaziou 6631 (n.v.); Brasil, Rio de Janeiro, Serra da Bica, Cascadura, 10/XII/1882, fl. masc., Glaziou 14046 (isosíntipo: R!); Brasil, Rio de Janeiro, Widgren (n.v.), comb. nov.

Ardisia warmingii (Mez) Bernacci \& JungMendaçolli. Stylogyne warmingii Mez in A. Engler, Pflanzenr. 9: 278. 1902. Tipo: Brasil, São Paulo, Espírito Santo do Pinhal, 25/XI/1895, fl., Campos-Novaes in CGG 3304 (isosíntipo: SP!); Brasil, São Paulo, São José do Rio Pardo, 1/X/ 1889, fl., A. Loefgren in CGG 1434 (isosíntipo: SP!); Brasil, Minas Gerais, Lagoa Santa, 23/XII/ 1863, Warming 522 (n.v.), comb. nov.

Através das foto de Stylogyne depauperata (Glaziou 4073 - F) e de $S$. pauciflora (Sellow 472 - F) foi possível observar que, nas ilustrações que acompanham as exsicatas, existem identificações como Ardisia depauperata e A. pauciflora, respectivamente; entretanto, através de consulta ao Field Museum of Natural Hystory não foi possível determinar quem fez as identificações e ilustrações (Laura Torres - comunicação pessoal).

Devido ao tipo de inflorescência em comum, panícula ou cima tirsóide, Stylogyne foi circunscrito em Ardisia, por Miquel (1856). Posteriormente, Mez (1902) sugeriu que Ardisia e Stylogyne pertenceriam a tribos distintas, devido à placentação em mais de uma série que caracterizaria o primeiro, e a placentação unisseriada que caracterizaria o segundo.

Entretanto, Ricketson \& Pipoly (1997) indicaram que espécies de Stylogyne podem ter 
óvulos em mais de uma série (bisseriados), apesar de assinalarem que se trata de evento raro. Por outro lado, Pipoly (1996) e Ricketson \& Pipoly (1997) reconheceram casos, em Ardisia, em que os óvulos são poucos e distribuídos em hélice, situação em que consideraram a placenta como plurisseriada, mas simulando condição unisseriada.

Mez (1902) descreveu Ardisia venosa Mast. como tendo óvulos em duas séries, entretanto em nossas observações verificou-se a existência de uma única série. Ståhl (1997) também observou uma única série de óvulos em $A$. venosa. Quando os óvulos são grandes em relação ao tamanho da placenta, conforme observado em $A$. crispa (Tab. 1), uma possível distribuição bisseriada dos óvulos, ou ao menos em hélice, é muito pouco evidente e não pode ser observada com segurança. Na ilustração desta espécie, apresentada por Mez (1902), os óvulos são pequenos em relação ao tamanho da placenta e distribuem-se com pequeno desnível entre si. As variações na placentação relatadas na literatura e aquelas observadas no presente estudo evidenciam que revisões amplas são necessárias, não só para a tribo Ardisiae, como sugerido por Pipoly (1996), mas para a família Myrsinaceae como um todo, reconsiderando a divisão em tribos. Anderberg \& Ståhl (1995) apontaram afinidades entre Ardisia e Stylogyne. Através de análise cladística, com base em observações morfológicas e anatômicas, Ståhl (1997) evidenciou afinidades entre Ardisia e Stylogyne e concluiu que a condição pauciovulada evoluiu a partir de uma condição multiovulada, independentemente, em mais de uma ocasião na subfamília Myrsinoideae (Myrsinaceae).

Além de diferenças na placentação e na disposição dos lobos da corola e cálice, Ricketson \& Pipoly (1997) apontaram diferenças nos ramos da inflorescência, que seriam opacos em Ardisia e róseos a hialinos e translúcidos em Stylogyne. Entretanto, verificou-se que em Ardisia compressa (Bernacci 2659 - IAC) e
Stylogyne ambigua (Bernacci 1282 - HRCB, IAC, SP; Bernacci 24502 - ESA, UEC e Schuster s.n. - IAC 37817), os ramos da inflorescência eram róseo-avermelhados, mas não translúcidos, no material vivo, e ficaram acastanhados, no material herborizado. Em todas os outros espécimes e nas outras espécies analisadas (Tab. 1), os ramos estavam acastanhados no material herborizado, indicando que o caráter também não é apropriado para distinguir Stylogyne de Ardisia.

O número de lobos da corola não é constante entre as espécies de Ardisia (ou Stylogyne) e pode variar dentro de uma espécie (por ex. $S$. depauperata - Tab. 1), tal como já observado por Mez (1902).

Ainda como resultado da presente análise, observou-se que A. guyanensis é bastante polimórfica, não só em relação a diferenças na placentação, como também em relação a outros caracteres. Smith \& Downs (1957) constataram variações em relação às glândulas nas pétalas. A presença ou ausência de glândulas nas pétalas havia sido considerada como caráter distintivo entre $A$. catharinensis $\mathrm{Mez}$ e $A$. guyanensis e utilizada na chave por Mez (1902). Em função de suas observações, Smith \& Downs (1957) sinonimizaram A. catharinensis em A. guyanensis.

No presente estudo observou-se que, no Estado de São Paulo, a presença de glândulas nas pétalas de $A$. guyanensis nem sempre é nítida, enquanto nos materiais da Ilha do Cardoso estas glândulas são atropurpúreas e facilmente visíveis, como já descritas por Jung-Mendaçolli \& Bernacci (1997); em exemplares de outras localidades as glândulas podem ser translúcidas (Franciosi et al. 2 - ESA, IAC, SP, UEC, Ivanauskas 505 - ESA, IAC) ou as pétalas podem ser muito escuras e as glândulas não são visíveis (Brade 7925 e 8180 - R), podendo ser interpretadas como ausentes ou efetivamente o são.

Variações em relação à presença de pilosidade nas folhas de $A$. guyanensis têm sido 
constatadas. Ao contrário do referido para a espécie por Mez (1902), Smith \& Downs (1957) observaram, para espécimes de Santa Catarina, que as folhas eram glabras ou subglabras. JungMendaçolli \& Bernacci (1997) observaram que, na Ilha do Cardoso, as folhas eram glabras. No presente estudo, observou-se que o material Black 48-3324 (IAC) é glabro, exceto pela presença de pilosidade ferrugíneo-lepidota decídua nos ramos da inflorescência e cálice.

Ardisia handroi Toledo ex Handro (1969) foi descrita e caracterizada por apresentar folhas inteiras, característica, no entanto, que pode ocorrer em A. guyanensis. Em relação a outros caracteres, $A$. handroi normalmente encontrase dentro dos limites de variação de $A$. guyanensis. Entretanto, A. handroi foi descrita como dióica, característica não descrita para Ardisia, mas possível para Stylogyne, de acordo com Mez (1902). Para a Ilha do Cardoso, Jung-Mendaçolli \& Bernacci (1997) sugeriram que A. guyanensis apresentasse protandria, pois observaram botões em que os estames já estavam desenvolvidos, enquanto o pistilo era rudimentar. Entretanto, com a ampliação da amostragem foi possível verificar a ocorrência de plantas polígamas (Melo 982 \& Correa IAC, SP - material considerado como protândrico, por Jung-Mendaçolli \& Bernacci 1997) ou dióicas (Moraes 827 \& Capelari Jr. - ESA, IAC; Kirizawa 2380 \& Silva - IAC, SP) que apresentam flores em que o pistilo não se desenvolve (pistilódio) ou, mesmo, é totalmente ausente (Shepherd et al. 10472 - UEC).

A placentação unisseriada em $A$. guyanensis (Tab. 1), ao menos aparentemente, e a existência de plantas dióicas podem levar à identificação dos espécimes como pertencentes ao gênero Stylogyne. Mez (1902) utilizou o tipo de margem foliar e o grau de ramificação das inflorescências para distinguir $S$. sellowiana $\mathrm{Mez}$ e $S$. pauciflora Mez, referidas como ocorrentes em São Paulo. Entretanto, estas características mostraram-se variáveis e não correlacionadas entre si ou com outras características da planta, nos materiais examinados. A grande variação observada em Ardisia guyanensis faz supor que A. catharinensis, A. handroi, Stylogyne pauciflora e $S$. sellowiana tenham sido descritas considerando-se espécimes extremos em um ou outro aspecto de A. guyanensis. A análise de maior amostragem (49 espécimes) mostrou a existência de muitos materiais com características intermediárias, indicando a possibilidade de sinonimização destes nomes em $A$. guyanensis. Uma conclusão definitiva, entretanto, demanda a análise dos materiais-tipo, o que não foi possível, até o momento.

\section{Agradecimentos}

Aos curadores dos herbários BAUR, BOTU, ESA, F, FCAB, HB, HRCB, IAC, ISA, R, RB, SP, SPF, SPFR, SPSF, UEC e UNBA; à Laura Torres (F), pela atenção e grande colaboração em relação aos materiais e às informações solicitadas; a Fábio Vitta (UNICAMP), pelo auxílio em relação ao Abstract; à Daniela Zappi (K) e M. Fátima Freitas (UNESP), pelos auxílios em relação à bibliografia.

\section{Referências bibliográficas}

Anderberg, A. A. \& Ståhl, B. 1995. Phylogenetic interrelationships in the order Primulales, with special emphasis on the family circumscriptions. Canadian Journal of Botany 73: 1699-4730.

Handro, O. 1969. Plantas novas do Brasil: 3. Loefgrenia 39: $1-5$.

Jung-Mendaçolli, S. L. \& Bernacci, L. C. 1997. Flora Fanerogâmica da Ilha do Cardoso (São Paulo, Brasil): Myrsinaceae. Flora Fanerogâmica da Ilha do Cardoso 5: 81-98.

Mabberley, D. J. 1987. The plant book. Cambridge University, Cambridge.

Mez, C. 1902. Myrsinaceae. Das Planzenreich (9): 1437.

Miquel, F. A. G. 1856. Myrsineae. In C. F. P. Martius, A. G. Eichler \& I. Urban (Eds.), Flora Brasiliensis 10: 269-324, t. 24-59.

Pipoly, J. J. 1991. Notas sobre el genero Ardisia Swartz en Colombia. Caldasia 16(78): 277-284.

Pipoly, J. J. 1996. New species of Ardisia (Myrsinaceae) from Ecuador and Peru. Sida 17(2): 445-458. 
Ricketson, J. M. \& Pipoly, J. J. 1997. Nomenclatural notes and a synopsis of Mesoamerican Stylogyne (Myrsinaceae). Sida 17(3): 591-597.

Smith, L. B. \& Downs, R. J. 1957. Resumo preliminar das Mirsináceas de Santa Catarina. Sellowia 8: 237-248.
Ståhl, B. 1997. The relationships of Heberdenia bahamensis and H. peduliflora (Myrsinaceae). Botanical Journal of the Linnean Society 122: 315-333. 